\title{
LA METODOLOGÍA DE LA ENSEÑANZA PARA LA COMPRENSIÓN EN LA MEJORA DEL RENDIMIENTO ACADÉMICO DE LOS ESTUDIANTES DE LA EDUCACIÓN SUPERIOR: EL CASO DE LA UNIVERSIDAD CATÓLICA DE EL SALVADOR ${ }^{1}$
}

\author{
THE TEACHING FOR UNDERSTANDING METHOD AND ITS ROLE IN IMPROVING HIGHER \\ EDUCATION STUDENTS' PERFORMANCE: THE CASE OF UNIVERSIDAD CATÓLICA DE EL SALVADOR \\ A METODOLOGIA DO ENSINO PARA O ENTENDIMENTO NA MELHORA DO RENDIMENTO \\ ACADÉMICO DOS ESTUDANTES DA EDUCAÇÃO SUPERIOR: O CASO DA UNIVERSIDAD \\ CATÓLICA DE EL SALVADOR \\ Antonia Larissa Hernández Monterrosa²
}

Fecha de recibido: Agosto 5 de 2017 | Fecha de aprobado: Octubre 5 de 2017

\begin{abstract}
Resumen
El modelo de enseñanza para la comprensión $(\mathrm{EPC})$ constituye un enfoque de enseñanza-aprendizaje basado en competencias y desempeños. Este se desarrolló en la década de los noventa en el Proyecto Zero, en la Universidad de Harvard. La metodología para realizar la investigación fue de tipo descriptivo; la muestra fue por conveniencia: participaron 40 docentes y 20 estudiantes de la universidad. Para la recolección de la información se formaron focus groups y se usó la técnica de la entrevista a profundidad, con su respectiva guía de entrevista. El principal resultado es que los docentes de la universidad desconocen de forma técnica el concepto de la enseñanza para la comprensión; sin embargo, utilizan diferentes estrategias para que los estudiantes asimilen los contenidos establecidos en los programas de estudio. Se concluye que la comprensión es un proceso de nivel intelectual avanzado que estimula al discente a formar pensamiento organizado y crítico ante la realidad para resolver problemas de forma creativa y eficiente.
\end{abstract}

Palabras clave: Didáctica, metodología, estrategias, comprensión, contenidos, aprendizaje.

1 Artículo de revisión.

2 Máster en Profesionalización de la Docencia Superior y Licenciatura en Ciencias de la Educación con especialidad en Educación Parvularia, Universidad Católica de El Salvador. Miembro de la red de investigadores del Observatorio de la Diversidad Religiosa y de las Culturas en América Latina y El Caribe. Correo electrónico: larissa.hernandez@catolica.edu.sv 


\section{Abstract}

The teaching for understanding method is a teaching and learning approach based on competencies and performance. It was developed in the nineties, in the framework of Project Zero (Harvard University). The researchers followed a descriptive methodology and used a convenience sampling. The sample was composed of 40 teachers and 20 students from the university. The authors gathered information through focus groups and in-depth interviews with their corresponding guide. The main finding is that teachers at this university are not familiar with the technical concept of teaching for understanding, although they apply different strategies for students to grasp the curriculum contents. The authors conclude that understanding is an advanced intellectual process that stimulates the learner to build organized and critical thinking towards reality, in order to solve problems creatively and efficiently.

Keywords: Didactics, methodology, strategies, comprehension, contents, learning.

\section{Resumo:}

0 modelo de ensino para o entendimento $(\mathrm{EPC})$ constitui um enfoque de ensino-aprendizagem baseada em concorrências e desempenhos. Este desenvolveu-se na década dos noventa no Projecto Zero, na Universidade de Harvard. A metodologia para realizar a investigação foi de tipo descritivo; a mostra foi por conveniência: participaram 40 docentes e 20 estudantes da Universidade. Para a coleta da informação formaram-se focus groups e usou-se a técnica da entrevista a profundidade, com sua respectiva guia de entrevista. A principal descoberta é que os docentes da Universidade desconhecem de forma técnica o conceito do ensino para o entendimento; no entanto, utilizam diferentes estratégias para que os estudantes assimilem os conteúdos estabelecidos nos programas de estudo. Conclui-se que o entendimento é um processo de nível intelectual avançado que estimula ao discente a formar pensamento organizado e crítico da realidade para resolver problemas de forma criativa e eficiente.

Palavras-chave: Didática, metodologia, estratégias, entendimento, conteúdos, aprendizagem. 


\section{INTRODUCCIÓN}

La actualización docente con respecto al área metodológica es importante ya que se requiere en gran medida para desarrollar eficazmente los contenidos. De esta forma habrá una mejor relación con la sociedad, puesto que representa un reto intelectual de trascendencia, responsabilidad humana y social, para la formación de las nuevas generaciones, en vista a un mejor desarrollo del país.

Actualmente, los docentes universitarios se encuentran con retos en las aulas, principalmente: las inteligencias múltiples, grupos numerosos, conducta, etc. Ante estas situaciones y otras más, el docente como agente principal de la educación tiene que prepararse para salir adelante con los estudiantes; él tiene que buscar formas de desempeñarse eficientemente en el aula y lograr que sus estudiantes comprendan los contenidos que se desarrollan en clase.

En este sentido es necesario buscar alternativas para mejorar el proceso educativo, por ejemplo, la enseñanza para la comprensión $(\mathrm{EpC}),{ }^{3}$ que es una estrategia pertinente, dinámica, reflexiva, de interacción entre maestros y estudiantes. Esta estrategia de la EpC se implementó en el año de 1979 por Howard Gardner y un grupo de colegas de la Universidad de Harvard, quienes comienzan una investigación sobre el potencial humano que da lugar a la formulación del Proyecto Zero, del cual fue cofundador David Perkins. El modelo de EpC tiene su origen en ese proyecto, teniendo como finalidad comprender y promover el aprendizaje, el pensamiento y la creatividad en las distintas disciplinas, instituciones y personas. La EpC funciona en doble dirección, una hacia el maestro, proporcionándole herramientas que enriquecen su trabajo, y otra hacia los estudiantes, a quienes les ofrece espacios de interacción con sus compañeros. Por lo tanto, la enseñanza para la comprensión requiere del concepto de enseñanza activa. Un docente activo está comprometido con el aprendizaje del estudiante a través del ejemplo, además de proporcionar explicaciones, estimular las inferencias y plantear situaciones problemáticas, para incentivar el establecimiento de patrones en el desarrollo del proceso de enseñanza y aprendizaje.

3 A partir de este apartado se hará referencia a la Enseñanza para la Comprensión a través de sus siglas, EpC.

\section{ANTECEDENTES}

La comprensión es un término que implica procesos cognitivos de alto nivel, esto implica emular a los demás o aplicar el conocimiento comprendido en la vida cotidiana. El ser humano tiene la capacidad de comprender el mundo que le rodea, pero ¿qué es lo que se comprende? Escobedo, Jaramillo y Bermúdez (2004) consideran que “(...) lo que se comprende es la realidad, o, por lo menos, una parte de ella; una parte de la realidad natural, de la realidad social o de la realidad histórica" (p. 529). Esto implica que cuando un estudiante ha asimilado los contenidos los puede reproducir, explicar o demostrar la habilidad que ha adquirido durante la asignatura.

Por ello, los docentes deben buscar metodologías, estrategias o técnicas para que sus estudiantes comprendan los contenidos que se les enseña. Una de estas técnicas es el programa de enseñanza para la compresión, que tiene como objetivo primordial que “(...) los estudiantes comprendan lo que se les está enseñando y lo puedan adaptar, utilizar y poner en práctica en el aula y fuera de ella, que les permita ser crítico y reflexivo ante los hechos y sucesos del acontecer diario" (Ayones \& Silvera, 2014, p. 12). Es decir, que tengan la capacidad de afrontar los problemas que resultan en el trabajo y tomar buenas decisiones con base en la teoría aprendida en los años de universidad.

Es de agregar que la metodología de la enseñanza para la comprensión busca provocar en los estudiantes el "despertar un interés reflexivo hacia las materias que están aprendiendo y ayudarlos a establecer relaciones entre su vida y la asignatura, entre los principios y la práctica, entre el pasado y el presente y entre el presente y el futuro" (Clavel \& Torres, 2010 , p. 2); es decir, busca hacer una correlación del contexto en el que vive el estudiante. En consecuencia, los docentes deben estar a la vanguardia de las tendencias en la práctica de la enseñanza, de tal manera que se asuma un compromiso para despertar en los estudiantes un interés reflexivo hacia las materias que están aprendiendo y para ayudarlos a establecer relaciones entre su vida y la asignatura, entre los principios y la práctica (Blythe, 2010).

En varias ocasiones, después de haber desarrollado la clase los estudiantes tienen un sin fín de preguntas, por ejemplo: ¿cómo vamos a aplicar este tema en nuestra vida? ¿Qué se debe hacer para desarrollar la guía? ¿Qué quiere el licenciado que hagamos? ¿Cómo hizo para obtener ese resultado? 0 manifiestan entre sus compañeros: "No entiendo lo que el 
docente quiere decir". Igualmente, en las evaluaciones que se realizan en cada período se evidencia que por la deficiente interpretación o comprensión de los contenidos sus respuestas no son pertinentes o no se ajustan a lo que se está preguntando. Ante estas situaciones el docente se frustra y se preocupa; hasta puede cuestionarse si en realidad está haciendo bien su trabajo. No obstante, no debe caer en esa situación ni darse por vencido para que sus estudiantes asimilen los contenidos de la clase; por el contrario, debe buscar estrategias para que sus estudiantes lleguen a comprender y en consecuencia asimilar los contenidos. Desde el planteamiento de Boix y Jackson (2011), existen cuatro características clave de la comprensión interdisciplinaria de calidad:

Primero, la comprensión interdisciplinaria tiene un propósito: los estudiantes examinan un tema para explicarlo o contar una historia sobre él de maneras que no serían posibles a través de una sola disciplina. En segundo lugar, la comprensión se basa en disciplinas: emplea conceptos, grandes ideas, métodos e idiomas de dos o más disciplinas de maneras precisas y flexibles. En tercer lugar, la comprensión interdisciplinaria es integradora: las persecuciones disciplinarias se integran para profundizar o complementar la comprensión. Y cuarto, la comprensión interdisciplinaria es reflexiva: los estudiantes reflexionan sobre la naturaleza del trabajo interdisciplinario y los límites de su propia comprensión. (p. 13).

Dicho lo anterior, los docentes de educación superior deben buscar metodologías por medio de las cuales sus estudiantes comprendan lo que se les está enseñando, porque muchas veces algunos solamente se limitan a desarrollar los contenidos establecidos en el programa de la asignatura sin tener en cuenta si los estudiantes comprendieron. Como docentes, es prioritario hacerse ciertas preguntas: ¿qué necesito que los estudiantes comprendan? ¿Cómo se pueden involucrar a los estudiantes activamente?

Actualmente existe una variedad de metodologías, técnicas y estrategias, pero en la investigación que se realizó se hizo énfasis es en la EpC porque evita que el proceso educativo se vuelva algo rutinario y permite a los estudiantes comprender y analizar el contexto que les rodea. Los docentes deben considerar que la metodología de la EpC se ocupa en desarrollar, según Baquero y Ruiz (2005): “La capacidad de resolver problemas, o de crear productos, que sean valiosos en uno más ambientes culturales (...) y que se expresa siempre en el contexto de tareas, disciplinas y ámbitos específicos" (p. 77). En este sentido, la EpC pretende que los estudiantes sean independientes y proactivos en las diferentes tareas educativas de acuerdo al área en la que se están especializando.

La EpC tiene elementos que guían el proceso de enseñanza aprendizaje. A continuación, se detallan:

Con respecto a los hilos conductores, son los que guían el aprendizaje disciplinar a largo plazo. Para Barrera y León (2009): “Evidencian el gran horizonte de la educación y muestran que entre más se comprende sobre algo, más en cuenta se tiene de lo que hace falta por comprender" (p.28). Son una guía que muestra al docente y al estudiante hacia dónde se quiere llegar y esforzarse por alcanzar la meta trazada.

Sobre los tópicos generadores, se conocen como un tema de cualquier índole, pero si se le agrega la palabra 'generador', lo que se fusiona como una forma de inventar en el estudiante un pensamiento creativo. Vasco, Bermúdez, Escobedo, Negret y León (2002) definen este concepto como un:

(...) enunciado de forma, por lo general, interrogativa, es decir, una pregunta que contiene en sí misma la polaridad de una paradoja, el poder de explicitar una insuficiencia o una contradicción en las formas de comprender la realidad que genera una amplia gama de hipótesis y nuevas preguntas (...) todo ello en búsqueda de un nuevo equilibrio. (p. 1).

Esta forma de interrogar a los estudiantes suscita procesos de análisis de los diferentes fenómenos que los rodean. Es importante sacarlos de la zona de comodidad para que enfrenten posibles situaciones que impliquen respuestas rápidas para solventarlas eficientemente.

Los desempeños de comprensión son actividades planificadas que buscan ir más allá, es decir, que los estudiantes no se sometan a una rutina o a la memorización. En este sentido, los desempeños de comprensión se definen como “(...) la forma en la que los estudiantes han asimilado los conocimientos y pueden utilizarlos de manera innovadora" (Blythe, 2010, p. 40). Esto los hace capaces de resolver cualquier eventualidad que surja en su vida estudiantil.

Asimismo, Fiore y Leymonié (2007) retoman el planteamiento de Perkins (1995), quien establece cuatro niveles de comprensión: 
- Nivel de contenido: en este nivel los estudiantes son capaces de realizar actividades de reproducción, tales como repetir, parafrasear, realizar procedimientos rutinarios.

- Nivel de la resolución de problemas: las actividades de comprensión están centradas en la ejemplificación y la aplicación.

- Nivel epistémico: las actividades de comprensión están centradas en generar interpretaciones y justificaciones de los hechos, datos, fenómenos que se estudian.

- Nivel de investigación: las actividades de comprensión se centran en el planteo y discusión de hipótesis, critica al conocimiento establecido, elaboración de conclusiones, etc. (pp. 2-3).

Finalmente, la valoración continua es un proceso que ayuda a apreciar el trabajo que se ha hecho con los estudiantes en el ciclo académico, permitiendo realizar en su debido momento retroalimentación y toma de decisiones para mejorar la manera en que se están desarrollando las clases o qué habilidades y destrezas necesitan los discentes afinar para desenvolverse eficientemente en la sociedad. Explicado lo anterior, la valoración continua se comprende como: “(...) una práctica que ofrece a los estudiantes una frecuente evaluación informativa en todo momento, no tanto con fines de calificación, sino para hacer avanzar su dominio de los desempeños que expresan su creciente comprensión" (Stone, 1999, p. 14). Asimismo, al evaluar se logra integrar dos elementos importantes en el proceso de aprendizaje. Bondy y Kendall (s. f.) consideran que "el desempeño y la retroalimentación es justamente lo que necesitan los alumnos cuando trabajan en el desarrollo de la comprensión de un tópico o concepto específico" ( $p$. 21). De esta manera los contenidos se asimilan mejor porque se verifica en las evaluaciones y prácticas que se realizan cotidianamente.

\section{OBJETIVOS}

\section{Objetivo general}

Analizar la formación de los docentes en la metodología de la enseñanza para la comprensión en la mejora del rendimiento académico de los estudiantes la educación superior, en el caso de la Universidad Católica de El Salvador.

\section{Objetivos específicos}

- Diagnosticar el conocimiento de los docentes sobre la metodología de la enseñanza para la comprensión en educación superior.

- Describir los elementos identificados que inciden en la enseñanza para la comprensión el proceso educativo de estudiantes universitarios.

- Identificar las estrategias didácticas para promover aprendizaje comprensivo en los estudiantes.

\section{FORMULACIÓN DEL PROBLEMA}

¿Cómo incide la metodología de la enseñanza para la comprensión en la mejora del rendimiento académico de los estudiantes la educación superior, en el caso de la Universidad Católica de El Salvador?

\section{DESARROLLO METODOLÓGICO}

La investigación se desarrolló con el método descriptivo. Se hizo una recopilación y presentación sistemática de datos obtenidos de los docentes y estudiantes sobre la enseñanza para la comprensión. La muestra para el estudio fue por conveniencia: se tomaron 40 docentes (10 de cada facultad) y 20 estudiantes ( 5 de cada facultad).

Para recolectar la información, como primer paso se convocó en días diferentes a los docentes de las facultades de la universidad, con la finalidad de poder manejar eficientemente la conversación entre ellos. El mismo procedimiento se hizo con los estudiantes, se utilizó la técnica de la entrevista a profundidad a través de focus groups y se usó una guía de entrevista.

Con respecto al análisis e interpretación de la información, esta fue sometida a un estudio sistemático a través de un análisis deductivo que permitió interpretar los datos en función de los objetivos planteados inicialmente en el estudio. 


\section{RESULTADOS}

\section{En relación con el conocimiento de los docentes sobre la metodología de la enseñanza para la comprensión en educación superior}

Desde la perspectiva de los docentes la enseñanza para la comprensión significa que los estudiantes "vean, analicen y comprendan los contenidos". Técnicamente, sobre este concepto el conocimiento es poco, pero evidentemente ellos buscan metodologías que faciliten la comprensión. Por ejemplo, para los profesores que enseñan materias numéricas les funciona la observación porque los estudiantes están atentos a cómo se resuelve un ejercicio; de esta forma, cuando se les asigna uno, y para que lo resuelvan sin dificultad, los docentes especialistas en esta área consideran que la matemática es, sobre todo, saber hacer, es una ciencia en la que el método claramente predomina sobre el contenido.

Los docentes coinciden en que las TIC ayudan a la innovación metodológica en el proceso de enseñanza aprendizaje, por ejemplo, mencionaron el uso de los celulares para buscar información, aunque enfatizaron en que se les debe dar a los estudiantes lineamientos para el empleo correcto de ellos, además de páginas adecuadas, y luego hacer las preguntas dirigidas, aunque también dijeron que no hay que dejar de lado lo tradicional porque hay elementos que son históricos y tienen que memorizarlos. Este hallazgo se fundamenta en el planteamiento de López, Jústiz y Cuenca (2013), quienes consideran que:

La significación de la memoria es más trascendente cuando se trata del estudio como actividad, en la cual se procesa y se construye de manera activa e independiente la información y el conocimiento. Es la memoria semántico-lógica la que posibilita la comprensión del significado del material que se estudia, la selección de las ideas más importantes, relacionarlas, organizarlas y estructurarlas para facilitar su conservación y actualización. (párr. 8).

Por tales razones se considera que esta es la forma de memoria que demanda mayor atención para mejorar su uso a partir de la diversidad de métodos, procedimientos, estrategias y técnicas.

Por otra parte, en el ámbito de la tecnología un docente comentó que últimamente utiliza los grupos de WhatsApp principalmente con los estudiantes de las carreras semipresenciales, ya que le permite estar en contacto rápido con ellos; sin embargo, enfatizó en que los grupos deben ser pequeños y que, en el caso contrario, con grupos grandes, no se puede usar esta opción.

Una de las docentes mencionó que la metodología del constructivismo y la teoría de Ausubel (el aprendizaje significativo) son alternativas para que el aprendizaje de los estudiantes sea efectivo y exista una mejor comprensión de los contenidos, visualizando que estos puedan aplicarse en la vida cotidiana. Además, otros docentes utilizan en las clases el trabajo colaborativo, ya que como metodología les funciona. En este sentido, la comprensión y los nuevos estándares exigen que los docentes seleccionen los contenidos curriculares más detenidamente de acuerdo a las necesidades de sus estudiantes, además de tener claros los propósitos o metas al momento de las evaluaciones, ya que estas deben estar acordes al desempeño del proceso de enseñanza-aprendizaje (Stone, 2005). Por lo tanto, todo docente debe considerar el contexto de los estudiantes con los que trabaja en un ciclo académico; de esta forma logrará orientarlos adecuadamente para que el aprendizaje sea efectivo.

Desde el punto de vista de los estudiantes es importante que los docentes mejoren en la práctica porque ellos se emocionan cuando ven el nombre de la asignatura, pero se frustran cuando no llegan a comprender los contenidos por la forma en que se imparte, ya que varios docentes solamente usan una metodología teórica, nada de práctica.

Los estudiantes de la Facultad de Empresariales manifestaron que se trabaja bastante con números y que, por lo tanto, las asignaturas en su mayoría son analíticas. Además, se realiza trabajo en equipo, se organizan ferias de productos, ya que les ayuda en la parte de cómo hacer promoción. Los estudiantes que formaron parte de este focus groups comentaron que muchos de los docentes los impulsan a que sean emprendedores y formar sus empresas.

En la Facultad de Ciencias de la Salud los estudiantes comentaron que los docentes tienen que aplicar metodologías prácticas, por ejemplo, en química, anatomía, laboratorio, bioquímica. Esto da a entender que es necesario que las asignaturas lleven el elemento práctico en un $50 \%$, lo que garantiza que los estudiantes asimilen lo teórico.

Una particularidad que mencionaron los estudiantes es que algunos docentes deben hablar de una 
manera comprensible, pues son de primer año y aún se están acostumbrando al ritmo de la universidad. Además, consideran que los docentes deben mostrar más ejemplos de los temas desarrollados en clase y explicar sobre los materiales para utilizar en los trabajos. Este aspecto que mencionan los estudiantes es hasta cierto punto comprensible porque a nivel de educación básica y media ellos aún no dimensionan las responsabilidades de un profesional. Probablemente, ese fenómeno se manifieste porque igual las exigencias no son muchas en esos niveles.

Otro de los hallazgos fue que en grupo de universitarios de tercer año de la Facultad de Ciencias y Humanidades considera que los catedráticos que les han impartido asignaturas hacen muy bien su trabajo, son dinámicos y siempre explican de modo que puedan comprender. Consideran que las asignaturas cursadas despiertan la creatividad. Esta idea la apoya el planteamiento de Brunner (citado en Perkins, 1991). Él escribió sobre el poder del sistema cognitivo humano específicamente: "Cuando las personas van más allá de la información dada (de manera razonable), entonces reconocemos que entienden" (p. 5). En este sentido, se puede corroborar a través de las acciones cotidianas que los estudiantes comprendieron la utilidad de los contenidos.

\section{Sobre elementos identificados que inciden en la enseñanza para la comprensión el proceso educativo de estudiantes universitarios}

En este caso se encontró que los docentes coinciden acerca de que las clases tienen que estar impregnadas de la realidad, de tal forma que los estudiantes puedan relacionar la teoría y la práctica. Así, algunos de los docentes implementan visitas técnicas, por ejemplo, ir a fincas, laboratorios, prácticas en escuelas y clínicas. Estas inspecciones, viéndolas desde el punto de vista de la EpC, se relacionan directamente con los desempeños de comprensión, los cuales son actividades planificadas que buscan ir más allá, es decir, que los estudiantes no se sometan a una rutina o a la memorización porque, aunque es necesario hacer uso de la memoria, es más importante comprender los contenidos. Con este elemento se busca que los estudiantes sean capaces de ejecutar una acción, cuando ellos pueden realizarlas es porque han comprendido las indicaciones. En este sentido, los desempeños de comprensión se definen como "(...) la forma en la que los estudiantes han asimilado los conocimientos y pueden utilizarlos de manera innovadora" (Blythe, 2010, p. 40).
Por otra parte, los docentes procuran que los estudiantes desarrollen la creatividad. La creación de blogs es una alternativa según una docente que imparte asignaturas en la carrera de Licenciatura en Inglés, además, de combinarlos con multimedia como producto y al final de la materia los estudiantes exponen sus creaciones. Una docente utiliza los organizadores gráficos en 3D, lo que les ayuda a los estudiantes a construir el material y conceptos para que los internalicen; asimismo, les sirve para ayudarse unos con otros, es decir, que busca que entre los estudiantes exista el trabajo colaborativo que sirve para reforzar entre compañeros los contenidos.

Otros elementos que inciden en la comprensión de los estudiantes son los análisis de casos y reflexiones porque esto lo pueden relacionar con la vida cotidiana. Definitivamente, en estos dos ejemplos los estudiantes desarrollan el pensamiento analítico, sintético y evaluativo de la realidad. Además, desarrollan el pensamiento crítico que facilita no solo la integración de los conocimientos de la materia, sino que también ayuda al alumno a generar y fomentar el trabajo en equipo y la toma de decisiones, así como la innovación y la creatividad.

Una docente comentó que en las clases le funciona una técnica de la gamificación esta consiste en que el aprendizaje se traslada la mecánica de los juegos al ámbito educativo-profesional con el fin de conseguir mejores resultados, ya sea para absorber mejor algunos conocimientos, perfeccionar alguna habilidad, o bien recompensar acciones concretas, entre otros muchos objetivos.

Otros elementos que los docentes utilizan en sus cátedras son subir videos en la plataforma Moodle para que los estudiantes los vean y luego los analicen con sus compañeros a través de un foro. Además, hacer investigaciones en empresas porque esto a los estudiantes les gusta. Esto sucede específicamente en el caso de Empresariales porque preguntan cómo se desencadenan situaciones particularmente en el área económica del país, cómo afecta el pago de bienes y servicios, los impuestos sobre la renta, el producto interno bruto y otros.

Por otra parte, desde la perspectiva de los estudiantes sobre la metodología que utilizan los profesores, consideran que es variada, por ejemplo, en clases se presentan imágenes para entender conceptos o se hacen mapas conceptuales, la plataforma Moodle se usa para subir contenidos, videos y a veces subir tareas. Los estudiantes comentaron que algunos profesores hacen uso de Facebook ya que a veces la 
plataforma falla y, entonces, tienen que recurrir a esta red social para subir tareas. En el caso de las clases de inglés, el uso de videos y audios ayuda enriquecer el vocabulario. Actualmente, las nuevas generaciones se movilizan a través de las redes tecnológicas de comunicación; estas se han convertido en una herramienta que permite el aprendizaje colaborativo e involucra espacios de intercambio de información que fomentan la cooperación.

Sobre estrategias didácticas para promover aprendizaje comprensivo en los estudiantes

Desde la perspectiva de los docentes, las estrategias didácticas van a depender del grupo de estudiantes y de la materia que se imparte. Esto, porque a que a veces tienen grupos numerosos. Además, la edad influye bastante, principalmente los que son de primer año y que aún tienen actitudes de adolescentes; por el contrario, los de cuarto y quinto año de carrera ya han madurado su pensamiento y es más fácil trabajar los contenidos.

Los profesores que trabajan en Ciencias Empresariales mencionaron que les funciona trabajar con casos prácticos de empresas. Por ejemplo, en el plan de marketing, y se le da un seguimiento paso a paso de lo que el estudiante va plasmando con la finalidad de que exista calidad y comprensión del trabajo que se hace en una empresa.

Un docente expresó que durante el desarrollo de la clase tienen que elaborar un portafolio con temas que les interesan o los temas que no han quedado claros, lo que a su vez le sirve al estudiante como una realimentación de la asignatura.

Los docentes manifestaron que es importante motivar a los estudiantes a que participen en debates porque ayuda a desarrollar la capacidad de análisis crítico y argumentación y, de esta forma, comprenden lo expuesto en clase. En virtud de esto, los estudiantes se hacen capaces de resolver cualquier eventualidad que surja en su formación profesional, ayudan a construir y demostrar su comprensión, brindando tanto a él como al docente la visión de cómo su comprensión evoluciona y se desarrolla ante nuevas situaciones, lo que hace visible el pensamiento del estudiante.

En el área de Agronomía, una estrategia importante es realizar visitas de campo, porque para el estudiante, resulta más significativo hacer los procedimientos en los laboratorios de biología, botánica, fisiología vegetal, etc. Es necesario que se planifique que $50 \%$ de la clase sea práctico y el otro $50 \%$ sea teoría.

En la opinión de los estudiantes, las estrategias didácticas que los docentes usan los fomentan a trabajar en equipo, por ejemplo, cuando son eventos en los que tienen que hacer presupuestos e inversiones en cuanto a productividad y promover los productos. En este sentido, estas actividades son vivenciales en esta área.

En la Facultad de Ciencias y Humanidades los docentes motivan a la reflexión, principalmente en las asignaturas humanísticas, en cada clase, a través de una serie de preguntas o estudios de clase.

Al comparar este hallazgo con la EpC se hace alusión a los desempeños de comprensión retomados por Mejía (2011) de Stone (1999), los cuales “(...) se caracterizan por ser acciones con mucha reflexión; estos requieren que los estudiantes demuestren su comprensión haciendo visible el pensamiento que sustenta sus acciones, tanto para el maestro como para los otros y para sí mismo" (p. 23). Los estudiantes pueden, por ejemplo, escribir ensayos, crear historias, realizar investigaciones, exponer, etc. Esto lleva a la reflexión a los docentes universitarios en el sentido de que deben ayudar a los estudiantes a desarrollar habilidades, pero se recomienda que puedan ponerse en práctica en el contexto en el que viven.

\section{CONCLUSIONES}

En la cotidianidad de las aulas universitarias se observan estudiantes con ciertas particularidades, lo que exige a los profesores diseñar e implementar estrategias didácticas que les permitan aproximarse a ellos, a sus diferentes estilos de aprendizaje, motivaciones y aspiraciones, a sus fortalezas, pero también a los aspectos que necesitan ser mejorados para que puedan avanzar en sus procesos de formación.

Al diagnosticar el conocimiento de los docentes sobre la metodología de la enseñanza para la comprensión en educación superior se pudo determinar que el procedimiento, como lo plantean en la Universidad de Harvard es desconocido. Sin embargo, los docentes de la universidad buscan alternativas por medio de las cuales los estudiantes asimilen los contenidos del programa de estudio. 
Con respecto a los elementos identificados que inciden en la enseñanza para la comprensión en el proceso educativo de estudiantes universitarios los docentes echan mano de lo que conocen metodológicamente hablando, es decir, del trabajo en equipo, aprendizaje significativo, uso de TIC, redes sociales, plataforma Moodle, etc. Si bien es cierto que estas actividades ayudan en el proceso educativo, es necesario que se busquen otras porque los estudiantes son cada vez más demandantes y como profesores es un compromiso capacitarse en materia de metodologías para la comprensión.

Sobre las estrategias didácticas para promover aprendizaje comprensivo en los estudiantes es necesario indagar más sobre este tópico, es necesario que estas estrategias generen en el estudiante pensamiento crítico, además de que puedan utilizarlas en el contexto en el que se desenvuelven y visualizar en un futuro cómo lo aprendido en la universidad les sirva en el ámbito laboral.

Con la información recopilada se determinó que los docentes están conscientes de la importancia de que los estudiantes realicen el proceso de comprensión, ya que esto incide en el rendimiento académico. Si bien los profesores conocen poco sobre el proceso de la EpC según el planteamiento de la Universidad de Harvard, se esfuerzan por que sus clases se desarrollen de la mejor forma para beneficio de sus estudiantes.

\section{RECOMENDACIONES}

La enseñanza para la comprensión es una alternativa, si se quiere ver de esa forma, para mejorar el rendimiento académico de los estudiantes. Es primordial que los docentes orienten enfáticamente el proceso de comprensión, ya que les permitirá a los estudiantes aislar, identificar y unir de forma coherente datos externos con los datos de que se disponen, es decir, los conocimientos previos.

La EpC busca que los alumnos sean pensadores críti$\cos$, que planteen y resuelvan problemas y que sean capaces de superar dificultades, ir más allá de la rutina y vivir productivamente en su propia sociedad. Las universidades deben comprometer a los estudiantes de manera más intensa y contar con la comprensión como su pieza central. El modelo EpC es un referente útil para innovar en las prácticas docentes, por su énfasis en el desarrollo de competencias y la evaluación por desempeños. Además, es un marco de trabajo pertinente para el contexto social y que permite una formación orientada al dominio disciplinario, con miras a formar profesionales capaces de dar respuesta a las exigencias laborales y las necesidades de la sociedad actual.

En definitiva, la Universidad Católica de El Salvador debe orientar a los docentes sobre esta estrategia con el fin de que se aplique en el proceso de enseñanza- aprendizaje. De esta manera se ayudará a que mejore el rendimiento académico de los estudiantes.

\section{REFERENCIAS}

Ayones, M., \& Silvera, P. (2014). Pertinencia de la práctica docente desde la enseñanza para la comprensión. [Tesis de maestría]. Universidad de la Costa Cuc, Barranquilla. Recuperado de http://repositorio.cuc.edu.co/xmlui/ bitstream/handle/11323/255/proyecto $\% 20$ de\%20grado\%2015\%20oct.pdf?sequence $=1$

Baquero, M. \& \&uiz, V. (2005). La enseñanza para la comprensión: una visión integradora de los fundamentos y estrategias de la enseñanza. Revista Actualidades Pedagógica, 46, 75-83. Recuperado de http://revistas.lasalle.edu. co/index.php/ap/article/view/1915/1781

Barrera, X., \& León, A. (2009). ¿De qué manera se diferencia el marco de la Enseñanza para la Comprensión de un enfoque tradicional? Ruta Maestra, 9, 26-32. Recuperado de http://www.santillana.com.co/rutamaestra/ediciones/ediciones.php

Blythe, D. (2010). La enseñanza para la comprensión: guía para el docente. Buenos Aires: Paidós.

Boix, M., \& Jackson, A. (2011). Educating for global competence: Preparing our youth to engage the world. New York: Asia Society. Recuperado de https://asiasociety.org/files/ book-globalcompetence.pdf

Bondy, E., \& Kendall, B. (S.f.). Valoración para la comprensión. Valoración continua. Curso en línea para educadores. Recuperado de http: / / live.v1.udesa.edu.ar/files/img/escuela-educacion/valoracion_continua.pdf

Clavel, J., \& Torres, J. (2010). La enseñanza para la comprensión como marco conceptual para el mejoramiento de la calidad educativa: la estrategia de la evaluación integrativa. Congreso Iberoamericano de Educación, Buenos 
Aires, Argentina. Recuperado de http:// www.chubut.edu.ar/descargas/secundaria/ congreso/ACCESO/R1857_Torres.pdf

Escobedo, H., Jaramillo, R., \& Bermúdez, A. (2004). Enseñanza para la comprensión. Educere, 8(27), 529-534. Universidad de los Andes Mérida. Recuperado de http://www.redalyc. org/pdf/356/35602712.pdf

Fiore, E., \& Leymonié, J. (2007). Planificaciones del aula que promueven la comprensión. Recuperado de http://maristas.org.mx/gestion/ web/articulos/planificaciones_aula_promueven_comprension.pdf

López, M., Jústiz, G. \& \& Cuenca, D. (2013). Métodos, procedimientos y estrategias para memorizar: reflexiones necesarias para la actividad de estudio eficiente. Humanidades Médicas, 13 (3), 805-824. Recuperado de http: / /www. humanidadesmedicas.sld.cu/index.php/hm/ article/view/405/306
Mejía, V. (2011). El marco para la Enseñanza para la Comprensión aplicado al aprendizaje del concepto de campo eléctrico en estudiantes de ingeniería en sistemas [Tesis de maestría]. Universidad Nacional de Colombia. Recuperado de http://www.bdigital.unal.edu. co/4382/1/04868123.2011.pdf

Perkins, D. (1991). Educating for insight. Educational Leadership, 49(2), 4-8. Recuperado de http: / / ascd.com/ASCD/pdf/journals/ed_ lead/el_199110_perkins.pdf

Stone, W. (1999). (Comp.). La Enseñanza para la Comprensión. Vinculación entre la investigación y la práctica. Buenos Aires: Paidós.

Vasco, C., Bermúdez, A., Escobedo, H., Negret, J., \& León, T. (2002). El concepto de tópico generador. Recuperado de http://live.v1.udesa.edu.ar/files/img/escuela-de-educacion/ topico.pdf 